\title{
Leflunomide versus cyclophosphamide in the induction treatment of proliferative lupus nephritis in Chinese patients: a randomized trial
}

\author{
Minfang Zhang ${ }^{1} \cdot$ Chaojun $\mathrm{Qi}^{1} \cdot$ Yan Zha ${ }^{2} \cdot$ Jian Chen ${ }^{3} \cdot$ Ping Luo ${ }^{4} \cdot$ Li Wang $^{5} \cdot$ Zhuxing Sun $^{6} \cdot$ Jianxin Wan ${ }^{7}$.

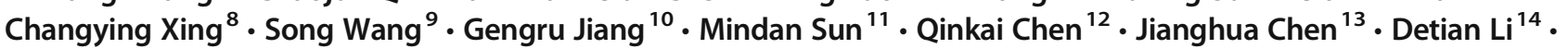 \\ Tianjun Guan ${ }^{15} \cdot$ Zhaohui Ni $^{1}$
}

Received: 21 January 2018 / Revised: 12 October 2018 / Accepted: 22 October 2018 / Published online: 13 November 2018

(C) The Author(s) 2018

\begin{abstract}
Objectives A prospective, multi-center, randomized controlled study was conducted to evaluate the efficacy and safety of a 24week course low-dose leflunomide combined with prednisone in the induction treatment of proliferative lupus nephritis in Chinese patients.

Method Patients $(n=100)$ with biopsy-proved proliferative lupus nephritis were enrolled in this study. They were randomized into two groups and received either leflunomide or cyclophosphamide in conjunction with prednisone for 24 weeks. Leflunomide was given orally with a loading dose of $40 \mathrm{mg} /$ day for 3 days followed by $20 \mathrm{mg} / \mathrm{day}$. Intravenous cyclophosphamide was administered monthly at a dosage of $0.8-1.0 \mathrm{~g}$. The primary efficacy outcome was the frequency of complete remission and partial remission at week 24 . The secondary outcomes included changes of urinary protein excretion, serum albumin, complement 3, anti-dsDNA antibody level, and systemic lupus erythematosus disease activity index (SLEDAI) after 24-week therapy. Results Of 100 patients, 48 received leflunomide combined with prednisone and other 52 received cyclophosphamide with concomitant prednisone. There were no statistically significant differences between groups in complete remission rate and partial remission rate. At week 24, 23\% of patients in the leflunomide group and $27 \%$ of patients in the cyclophosphamide group achieved complete remission $(P=0.64)$, while $56 \%$ of patients in the leflunomide group and $42 \%$ of patients in the cyclophosphamide group achieved partial remission at week $24(P=0.16)$. SLEDAI, serum albumin, complement 3 , anti-dsDNA antibody level, and urinary protein excretion improved significantly in both groups. No significant difference was seen in the changes of clinical parameters after therapy between the two groups. There was no significant difference in side effects in both groups. Conclusions Compared with cyclophosphamide, low-dose leflunomide in combination with prednisone showed both effectiveness and safety in the induction therapy of proliferative lupus nephritis in Chinese patients.
\end{abstract}

Keywords Efficacy $\cdot$ Leflunomide $\cdot$ Lupus nephritis $\cdot$ Prospective study

\section{Introduction}

Systemic lupus erythematosus (SLE) is an autoimmune disorder often involving multiple systems [1]. Lupus nephritis (LN), one of the most common complications of SLE, accounts for the most morbidity and mortality [2, 3]. Cyclophosphamide combined with glucocorticoids had been chosen as standard therapy in the induction treatment of lupus

Minfang Zhang and Chaojun Qi contributed equally to this work.

Zhaohui Ni

profnizh@126.com

Extended author information available on the last page of the article nephritis since the 1970s [4-6]. Recent randomized clinical trials showed that mycophenolate mofetil was as effective as cyclophosphamide in inducing remission of $\mathrm{LN}$ during the initial phase of therapy [7, 8]. Mycophenolate mofetil therefore is now commonly recommended as first-line therapy in LN [9-11]. In Asian patients, the response rate to intravenous cyclophosphamide was similar to that of mycophenolate mofetil [8], but nearly two times as many patients in the mycophenolate mofetil group withdrew because of adverse effects than the cyclophosphamide group in the largest clinical study and there were more deaths in the mycophenolate mofetil group [8]. Consequently, there is a need to find an alternative approach to LN treatment that is as effective as mycophenolate mofetil and with fewer severe adverse effects than mycophenolate mofetil. 
Leflunomide, an inhibitor of dihydroorotate dehydrogenase, has antiproliferative and anti-inflammatory actions by leading to a decrease of $\mathrm{T}$ cell and $\mathrm{B}$ cell. Leflunomide is a novel immunosuppressive agent used in the treatment of rheumatoid arthritis as a disease-modifying anti-rheumatic drug [12-14]. It has been proven that leflunomide is as effective as methotrexate in the treatment of rheumatoid arthritis and is well-tolerated [15].

There are some reports and studies indicating that leflunomide is safe, well-tolerated, and may be effective in SLE patients $[16,17]$. Leflunomide has also been explored for treatment of glomerulonephritis. It was reported to be effective in reducing the proteinuria in idiopathic membranous nephropathy and IgA nephropathy $[18,19]$. Several studies explored the effectiveness of leflunomide in the induction therapy of LN patients. The results of these studies indicated that the efficacy of leflunomide combined with glucocorticoid was comparable to cyclophosphamide [20,21]. A systemic review concluding 11 RCTs and a total of 254 patients evaluated the efficacy and safety of leflunomide compared to cyclophosphamide in adult patients with LN [22]. Results showed that leflunomide was superior to cyclophosphamide in achieving complete and total remission, but no difference in systemic lupus erythematosus disease activity index (SLEDAI) score was found between these two treatments. What's more, leflunomide was found to be safer than cyclophosphamide regarding adverse drug reactions (ADRs), including liver damage, alopecia, leukopenia, and infection. This meta-analysis of RCTs suggested that leflunomide is a promising therapy for LN treatment, primarily because of the comparable efficacy and favorable safety profile. The dosage of leflunomide given to patients was different, from 20 to $50 \mathrm{mg} /$ day. Some studies evaluated the efficacy of low-dose leflunomide (20 mg/day) in rheumatoid arthritis. However, study about the effectiveness of low-dose leflunomide in LN is sparse. We, therefore, undertook a randomized, prospective observational multi-center study to investigate the efficacy and safety profile of low-dose leflunomide compared with cyclophosphamide in the treatment of patients with biopsyproven proliferative lupus nephritis.

\section{Methods}

\section{Subjects}

Patients with LN were enrolled from 15 centers across China between November 2013 and November 2015. All patients were diagnosed as SLE according to the criteria of the American College of Rheumatology in 1997 [23], with a SLEDAI $\geq 8$. All of them had biopsy-proved diffuse proliferative or focal proliferative lupus nephritis (International Society of Nephrology/Renal Pathology Society, 2003 Type
IV A or A/C and Type III A or A/C) [24], with or without membranous nephropathy. The renal biopsy should be done within 6 months before randomization. Pathological activity index (AI) of every patient enrolled in this study was no less than 4. Patients who had received any immunosuppressant (such as cyclophosphamide, mycophenolate mofetil, cyclosporin, tacrolimus, leflunomide, methotrexate, and biologics) within the previous 1 month were excluded. Patients who had received high-dose steroids within the previous 1 month were also excluded. Other exclusion criteria were severe infection which need intravenous antibiotics, cerebral lupus, liver disease, malignant tumor, pregnancy, anticipated poor compliance with the protocol, and age $<18$. Patients with antiphospholipid syndrome or with positive antiphospholipid antibodies were also excluded in this study. The study was approved by the Chinese State Food and Drug Administration (2011L01968) and the ethics committees of Renji Hospital, School of Medicine, Shanghai Jiaotong University, and informed consent was obtained from each patient.

\section{Evaluation of renal biopsies}

Renal biopsy specimens were evaluated (immunofluorescence and light microscopy) by renal pathologists at each center, and categorized according to the ISN/RPS classification system. Those specimens were re-examined blindly by experienced pathologists in central laboratory to re-assess the diagnosis and evaluate the pathological indices of activity. Renal activity index (AI) scores were assessed according to Austin et al. [25].

\section{Therapeutic schedules}

The central randomization system provided by the department of epidemiology and health statistics, Nanjing Medical University, was used for randomization in the study. Investigators from each center enrolled participants. Immediately after informed consent was obtained, investigator logged into the central randomization system, obtained random number, and got the intervention plan. Thus, patients were randomized to the two treatment groups: leflunomide or cyclophosphamide. Leflunomide tablets (drugs were provided by Fujian Huitian Biological Pharmaceutical Co., ltd.) were given at an initial loading dose of $40 \mathrm{mg} /$ day for 3 days and followed by $20 \mathrm{mg} /$ day for 24 weeks. Intravenous cyclophosphamide was administered monthly for 6 months at a dose of $0.8-1.0 \mathrm{~g}$ monthly. All patients were given a dose of 0.8 $1.0 \mathrm{mg} / \mathrm{kg} / \mathrm{day}$ prednisone for 8 weeks. Dosage of prednisone was tapered by $5-10 \mathrm{mg}$ every 2 weeks until $20 \mathrm{mg} /$ day and then $2.5 \mathrm{mg}$ every 2 weeks until a maintenance dose of $10 \mathrm{mg} /$ day was reached. Prohibited medications include immunoglobulin and plasma exchange. If patients had been using 
hydroxychloroquine or ACE inhibitors before enrollment, these drugs could be used with the original dose during this study.

\section{Laboratory parameters}

The duration of the study was 24 weeks. During the follow-up period, patients were closely monitored for various clinical parameters and adverse events, every 2 weeks for the first month and then every 4 weeks for the remaining 20 weeks. The parameters included urinary protein excretion, urinalysis, white blood cell, hemoglobin, blood platelet count, serum creatinine (Scr), serum uric acid, blood urea nitrogen and liver functions, erythrocyte sediment rate, and C-reactive protein (CRP). Serum complement 3 (C3), complement 4 (C4), and anti-double-stranded DNA (anti-dsDNA) antibodies were also measured. Anti-nuclear antibody (ANA) and SLEDAI was evaluated at 0,12 , and 24 weeks. The titer of serum antibodies against double-stranded DNA (dsDNA) was determined by using radioimmunoassay (EUROIMMUN AG, Germany). ANA was detected by using an indirect immunofluorescence method. The normal range of anti-dsDNA antibody titers was less than $7 \mathrm{IU} / \mathrm{mL}$. The normal range of $\mathrm{C} 3$ and $\mathrm{C} 4$ was 0.9 $1.8 \mathrm{~g} / \mathrm{L}$ and $0.1-0.4 \mathrm{~g} / \mathrm{L}$. Estimated glomerular filtration rate (eGFR) was calculated using the modification of diet in renal disease (MDRD) formula.

\section{Endpoints of the study and definitions}

The primary endpoints were complete remission and partial remission at 24 weeks. The secondary endpoints were response of clinical parameters and side effects. Complete remission was defined as urinary protein excretion less than $0.3 \mathrm{~g} /$ day, with normal urinary sediment $(\mathrm{RBC}<5 / \mathrm{HP}$, WBC $<5 / \mathrm{HP}$ ), with normal serum albumin concentration and stable renal function (percent change in creatinine less than $15 \%$ from baseline). Partial remission was defined as any one of the following features: decrease of urinary protein excretion by more than $50 \%$, with a serum albumin level $\geq$ $30 \mathrm{~g} / \mathrm{L}$ and stable renal function. Treatment failure was defined as any of the following: increase of the serum creatinine level by $15 \%$ from baseline; urinary protein excretion $>3 \mathrm{~g} /$ $24 \mathrm{~h}$; failure of urinary protein excretion to improve by $50 \%$ with serum albumin level $<30 \mathrm{~g} / \mathrm{L}$.

\section{Statistical analysis}

The sample size necessary to detect a significant difference ( $\pi=80 \%, \alpha=0.05$, two-sided) was calculated to be 180 on the basis of 0.8 power according to Fisher's exact test. Normally distributed data were presented as mean and standard deviations, while non-normally distributed variables were expressed as median and quartile. The Student $T$ or
Mann-Whitney $U$ test was used to compare the variables between two groups when necessary. The comparison of all categorical variables such as frequency or percentage (\%) was performed using either the chi-square or Fisher's exact tests. Intention-to-treat analysis was performed on primary outcome. SPSS 19.0 software was used for statistical analysis. All statistical tests were two-sided and significance was defined as $P<0.05$.

\section{Results}

\section{Baseline characteristics of patients}

A total of 100 patients were included in the study, 48 in the leflunomide group and 52 in the cyclophosphamide group. The age of patients ranged from 21 to 61 years old. There were no significant differences in age, gender, serum creatinine, estimated glomerular filtration rate, urinary protein excretion, serum albumin, complement 3 level, and SLEDAI between the leflunomide group and cyclophosphamide group (Table 1). Renal pathological assessment showed that there was no significant difference in pathological type and AI score between the two groups (Table 1). Twenty-eight patients $(53.8 \%)$ in the cyclophosphamide group and 26 patients $(54.1 \%)$ in the leflunomide group had nephrotic range proteinuria. Seven patients $(13.5 \%)$ in the cyclophosphamide group and 12 patients $(25 \%)$ in the leflunomide group had elevated creatinine at baseline.

\section{Primary endpoints}

Of the 100 patients recruited, 90 patients finished the 24-week study. Ten patients did not complete this study (four in the leflunomide group and six in the cyclophosphamide group), and they were dealt with as treatment failure in the intentionto-treat analysis (Fig. 1). After 24-week treatment, no difference between the leflunomide and cyclophosphamide groups was addressed with respect to clinical outcome (Table 2). Moreover, we did not find significant difference in time to achieve partial remission between the leflunomide group and the cyclophosphamide group ( $11.0 \pm 6.5$ vs. $10.6 \pm 6.7$ weeks, $P=0.737)$.

\section{Change of various parameters}

Patients in both the cyclophosphamide and leflunomide groups showed significant improvement in disease activity markers. Urinary protein excretion, anti-dsDNA antibody level, and SLEDAI decreased significantly both in leflunomide and cyclophosphamide group. Serum albumin level and serum complement 3 level increased significantly in both groups (Table 3). Serial values for urinary protein excretion (Fig. 2a), 
Table 1 Baseline demographic characteristics of patients in two treatment arms

\begin{tabular}{llll}
\hline & LEF $(n=48)$ & CTX $(n=52)$ & $P$ value \\
\hline Gender (male/female) & $2 / 46$ & $3 / 49$ & 0.54 \\
Age (years) & $37.8 \pm 10.2$ & $39.6 \pm 10.1$ & 0.36 \\
Systolic blood pressure $(\mathrm{mmHg})$ & $128.6 \pm 14.3$ & $130.1 \pm 13.7$ & 0.61 \\
Diastolic blood pressure $(\mathrm{mmHg})$ & $79.0 \pm 11.1$ & $78.5 \pm 10.4$ & 0.82 \\
Proteinuria $(\mathrm{g} /$ day) & $3.89(1.46 \sim 5.48)$ & $3.63(2.01 \sim 6.90)$ & 0.36 \\
Serum albumin $(\mathrm{g} / \mathrm{L})$ & $26.69 \pm 8.70$ & $26.58 \pm 8.05$ & 0.95 \\
Serum creatinine $(\mu$ mol/L) & $63.4(51.6-104.8)$ & $71.3(56.8-84.0)$ & 0.87 \\
eGFR (mL/min/1.73 m $\left.{ }^{2}\right)$ & $103.3 \pm 42.6$ & $97.2 \pm 37.4$ & 0.45 \\
C3 $(\mathrm{g} / \mathrm{L})$ & $0.54 \pm 0.26$ & $0.46 \pm 0.27$ & 0.47 \\
C4 $(\mathrm{g} / \mathrm{L})$ & $0.08(0.06-0.14)$ & $0.07(0.04-0.11)$ & 0.06 \\
dsDNA $(\mathrm{IU} / \mathrm{L})$ & $29.42(7.95-94.90)$ & $42.43(13.38-98.88)$ & 0.07 \\
dsDNA $(n(\%))$ & $41(85.4)$ & $45(86.5)$ & 0.13 \\
ANA $(n(\%))$ & $44(91.7)$ & $46(88.5)$ & 0.21 \\
SLEDAI & $16.2 \pm 6.0$ & $15.4 \pm 6.5$ & 0.55 \\
Activity index & $8.6 \pm 2.6$ & $8.3 \pm 2.6$ & 0.41 \\
Pathological classification & & & \\
III & 9 & 9 & 0.83 \\
IV & 24 & 11 & \\
III+V & 5 & & \\
IV+V & 10 & & \\
\hline
\end{tabular}

Values are presented as means \pm standard deviation (SD) if the variables showed a normal distribution and as medians (IQR) if the variables did not show a normal distribution; $n(\%)$ was used for the categorical variables serum albumin (Fig. 2b), anti-dsDNA antibody concentration, serum complement 3 level, and SLEDAI were similar in the two groups (Fig. $3 \mathrm{a}-\mathrm{c}$ ). The magnitude of changes in serum albumin, 24-h urine excretion, serum complement 3, antidsDNA antibody level, and SLEDAI has no significant difference between the two treatment groups $(P>0.05)$.

\section{Adverse events}

There was no significant difference in the rate of adverse events between two groups (Table 4). The most common adverse event was infection in both groups. There were 18 episodes of infection in cyclophosphamide group and 16 episodes in patients treated with leflunomide. Upper respiratory infection was the most common infection in both groups. Herpes zoster happened in three patients in the cyclophosphamide group and in one patient in the leflunomide group. One patient who had herpes zoster in the cyclophosphamide group discontinued her treatment because of the adverse event. One patient died in the leflunomide group because of suicide. Though the suicide patient had achieved complete remission during the treatment period, she got depressive disorder after the disease onset. We also noticed that the incidence of abnormal liver function in the leflunomide group was higher than

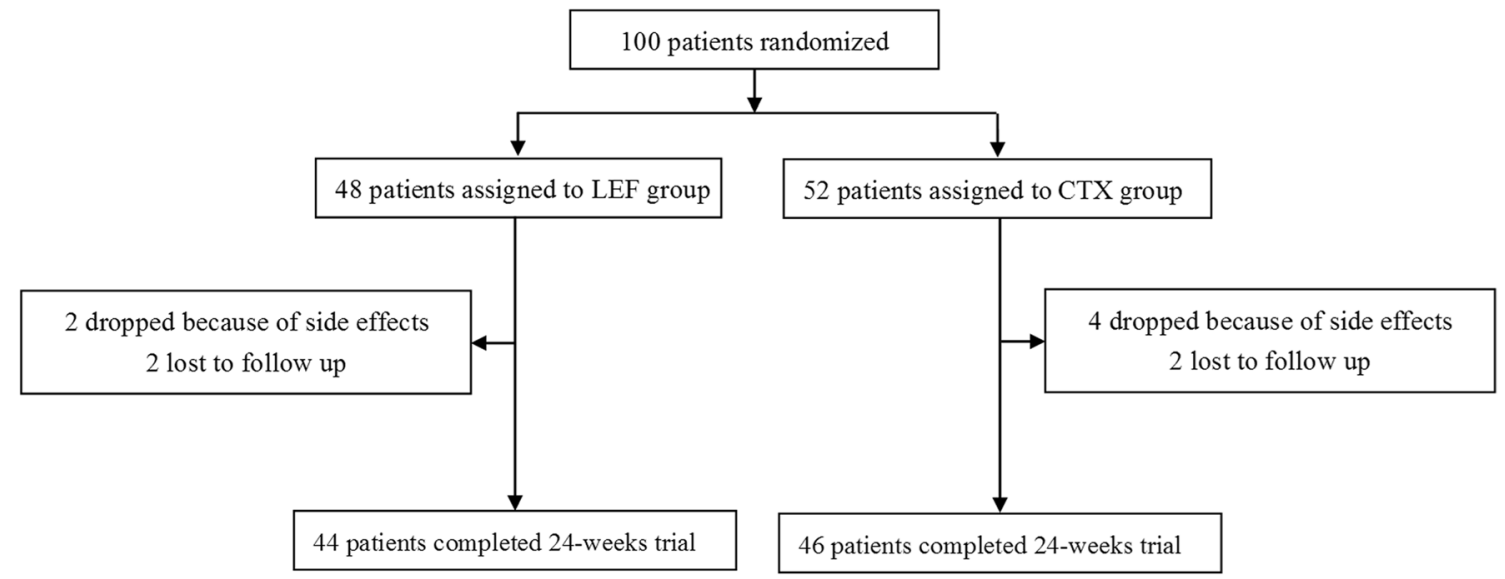

Fig. 1 Flowchart of patients enrolled and follow-up 
Table 2 Efficacy of leflunomide and cyclophosphamide treatment

\begin{tabular}{lllllll}
\hline & \multicolumn{2}{l}{$\operatorname{LEF}(n=48)$} & & \multicolumn{2}{c}{$\operatorname{CTX}(n=52)$} & \multirow{2}{*}{$P$ value } \\
\cline { 2 - 3 } & $n$ & $\%$ & & $n$ & $\%$ & \\
\hline Complete remission & 11 & 23 & & 14 & 27 & 0.64 \\
Partial remission & 27 & 56 & & 22 & 42 & 0.16 \\
Treatment failure & 10 & 21 & & 16 & 31 & 0.26 \\
\hline
\end{tabular}

that in the cyclophosphamide group, but the difference was not significant. All patients with impaired hepatic function were relieved after treatment with hepatoprotective drugs. Though there was no significant difference between the two groups, menorrhea and nausea happened less in the leflunomide group ( 0 episode and 1 episode) compared with cyclophosphamide group ( 2 episodes and 5 episodes).

\section{Discussion}

SLE is a relapsing autoimmune disease predominantly affecting women of childbearing age. Lupus nephritis is one of the most serious manifestations accounted for poor outcome. Since treatment requires long-time therapy, it is crucial not only to define medicines that are effective but minimize toxicity [3]. Currently, the main immunosuppressive regimens recommended for therapy of lupus nephritis include mycophenolate mofetil, cyclophosphamide, and azathioprine [10, 11], but adverse drug reactions such as infection, leucopenia, and liver damage occur in many patients leading to increased morbidity and mortality. Therefore, more effective drugs with favorable safety profile are needed.

Leflunomide, as an immunosuppressive regimen, was confirmed to be as effective as methotrexate and sulfasalazine in the treatment of rheumatoid arthritis in 1998. Since it was approved by the US Food and Drug Administration to be used in rheumatoid arthritis, leflunomide has been increasingly used in clinical applications [26]. As an immunosuppressive
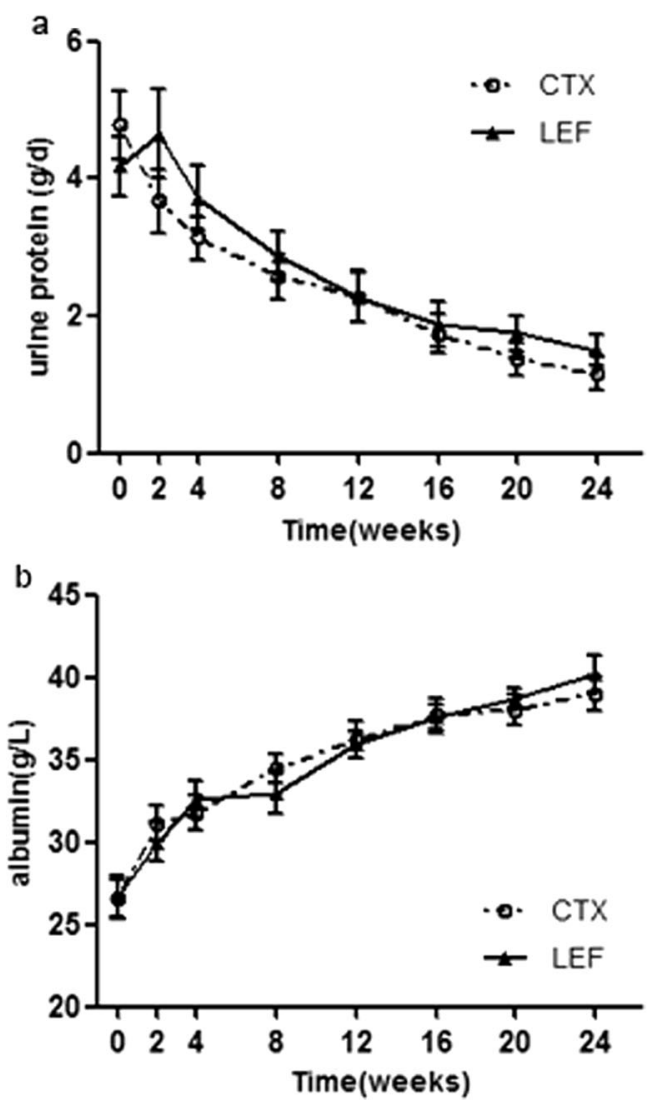

Fig. 2 Changes of mean $( \pm \mathrm{SD})$ urinary protein excretion and serum albumin in patients with proliferative lupus nephritis treated with leflunomide or cyclophosphamide for 24 weeks. In both groups, urinary protein excretion decreased gradually after treatment. The mean urinary protein excretion (a) decreased significantly after 8 weeks of therapy, and it remained significantly lower at the end of study $(P<0.05)$. The mean serum albumin (b) was significantly higher than the baseline value, and it remained significantly higher at the end of study $(P<0.05)$.

regimen, leflunomide has an inhibition effect on proliferation and activity of $\mathrm{B}$ and $\mathrm{T}$ cell, making it a reasonable candidate for treatment of LN.

Some experimental models [27] and clinical studies [17] have showed that leflunomide has an obvious ameliorative effect against SLE and LN. In chronic graft-versus-host

Table 3 Comparison of various parameters in patients before and after treatment in both groups

\begin{tabular}{|c|c|c|c|c|}
\hline & \multicolumn{2}{|l|}{ LEF } & \multicolumn{2}{|l|}{ CTX } \\
\hline & Before treatment & After treatment & Before treatment & After treatment \\
\hline Proteinuria (g/day) & $3.89(1.46 \sim 5.48)$ & $0.86(0.18 \sim 1.74)^{* *}$ & $3.63(2.01 \sim 6.90)$ & $0.50(0.16 \sim 1.18)^{* *}$ \\
\hline Serum albumin $(\mathrm{g} / \mathrm{L})$ & $26.69 \pm 8.70$ & $40.18 \pm 7.47 * *$ & $26.58 \pm 8.05$ & $39.01 \pm 7.05^{* *}$ \\
\hline Serum creatinine $(\mu \mathrm{mol} / \mathrm{L})$ & $63.4(51.6-104.8)$ & $60.0(53.1-69.5)$ & $71.3(56.8-84.0)$ & $61.3(53.9-75.0)$ \\
\hline Anti-dsDNA level (IU/L) & $29.42(7.95-94.90)$ & $9.49(7.29-19.45)^{* *}$ & $42.43(13.38-98.88)$ & $17.13(7.77-31.99)^{* *}$ \\
\hline $\mathrm{C} 3(\mathrm{~g} / \mathrm{L})$ & $0.54 \pm 0.26$ & $0.88 \pm 0.27 * *$ & $0.46 \pm 0.27$ & $0.82 \pm 0.34 * *$ \\
\hline SLEDAI & $16.2 \pm 6.0$ & $7.8 \pm 4.0^{* *}$ & $15.4 \pm 6.5$ & $7.1 \pm 4.0^{* *}$ \\
\hline
\end{tabular}

**Compared with the same parameters before treatment, $P<0.05$ 
Fig. 3 Changes of mean $( \pm \mathrm{SD})$ serum $\mathrm{C} 3$, anti-dsDNA antibody levels, and SLEDAI in lupus nephritis patients treated with leflunomide or

cyclophosphamide for 24 weeks. In both groups, serum antidsDNA antibody levels (a) and SLEDAI (c) were decreased significantly after 12 weeks of therapy, and remained significantly lower at the end of study $(P<0.05)$. Serum C3 levels (b) were increased significantly in both groups after 12 weeks of therapy and almost remained in normal level at the end of study $(P<0.05)$
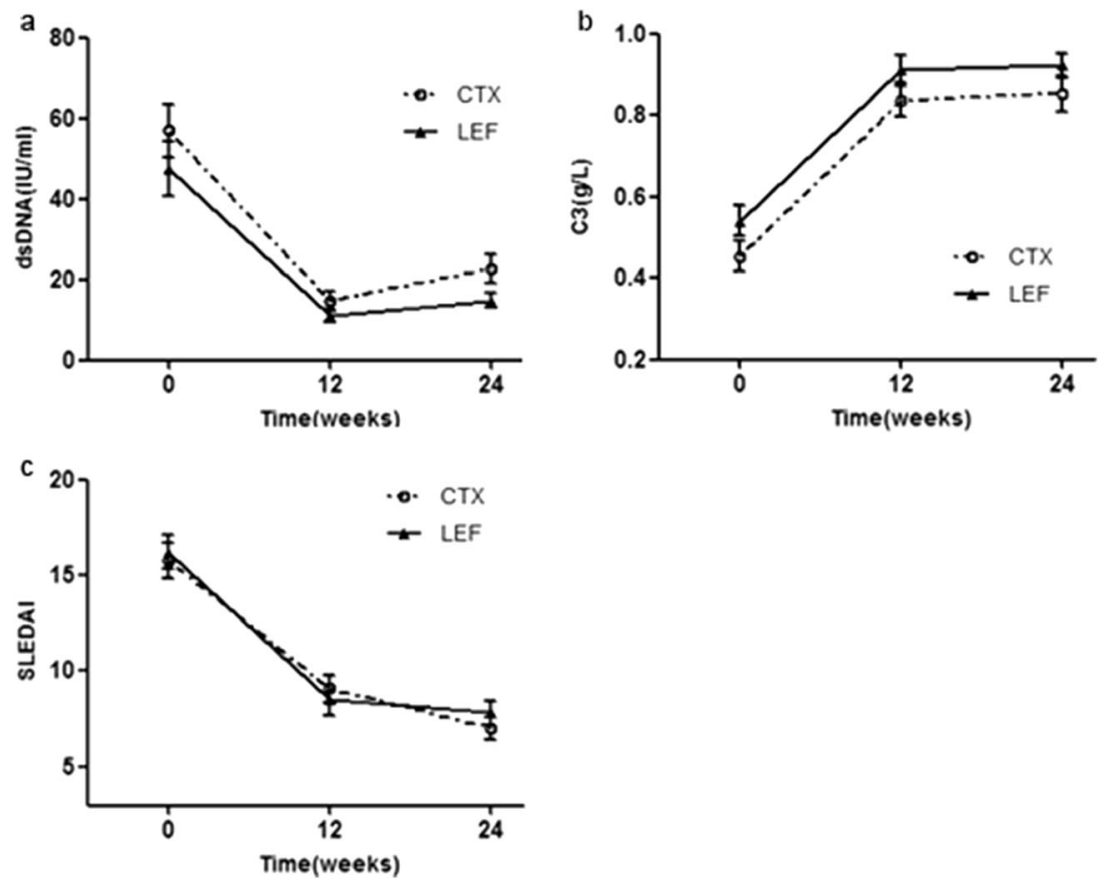

disease (cGVHD) mice model, compared with an untreated group, oral leflunomide administration for several weeks could increase survival, reduce levels of autoantibodies, and improve the histopathologic lesions by inhibition of TLR9 signaling pathway [28]. A771726, the active metabolite of leflunomide, was proven to have therapeutic effects in murine LN. A771726 could inhibit the development of $\mathrm{LN}$ and attenuate the generalized autoimmune features by

Table 4 Adverse events

\begin{tabular}{|c|c|c|c|c|c|}
\hline \multirow[t]{2}{*}{ Adverse effect } & \multicolumn{2}{|c|}{$\operatorname{LEF}(n=48)$} & \multicolumn{2}{|c|}{$\operatorname{CTX}(n=52)$} & \multirow[t]{2}{*}{$P$ value } \\
\hline & No. & $\%$ & No. & $\%$ & \\
\hline Infection & 16 & 33 & 18 & 35 & NS \\
\hline Upper respiratory infection & 9 & 19 & 9 & 19 & \\
\hline Pneumonia & 2 & 4 & 3 & 6 & \\
\hline Urinary tract infection & 4 & 8 & 3 & 6 & \\
\hline Herpes zoster & 1 & 2 & 3 & 6 & \\
\hline Leukopenia & 1 & 2 & 1 & 2 & NS \\
\hline Elevated liver enzymes & 9 & 18.8 & 5 & 9.6 & NS \\
\hline Hair loss & 1 & 2 & 2 & 4 & NS \\
\hline Amenorrhea & 0 & 0 & 2 & 4 & NS \\
\hline Diarrhea & 3 & 6 & 3 & 6 & NS \\
\hline Nausea & 1 & 2 & 1 & 2 & NS \\
\hline Hypertension & 5 & 10 & 1 & 2 & NS \\
\hline Headache & 1 & 2 & 3 & 6 & NS \\
\hline Pyknocardia & 2 & 4 & 4 & 8 & NS \\
\hline Death* & 1 & 2 & 0 & 1 & NS \\
\hline
\end{tabular}

*One patient died in the leflunomide group because of suicide augmenting iTregs which suppress pathogenic IL-17producing double negative $\mathrm{T}$ cells through an Aktdependent mechanism in MRL/lpr mouse. In 2004, the first double-blind, randomized, placebo-controlled clinical study conducted in Hong Kong showed the effectiveness of leflunomide in the treatment of SLE. Twelve patients were enrolled in this study. Six patients were randomized to receive leflunomide and the other six received placebo. All patients received the same dosage of prednisolone during the study. Patients in the leflunomide group were treated with leflunomide orally, while patients in the placebo groups received placebo instead. After treated with leflunomide (a loading dose of leflunomide $100 \mathrm{mg}$ daily for 3 days followed by leflunomide $20 \mathrm{mg}$ daily for the remainder of the study)-combined prednisolone, the SLEDAI score reduced significantly in the leflunomide group [17]. However, there was no significant difference between the two groups in the change of proteinuria. This might have resulted from only three of six patients treated with leflunomide with a mean proteinuria at $1 \mathrm{~g} /$ day that were recruited in this study. But after that, several studies showed that leflunomide was quite effective in the treatment of lupus nephritis [22]. Wang et al. found that leflunomide and cyclophosphamide had similar efficacy and safety in the treatment of proliferative $\mathrm{LN}$ in 2008 . A total of 110 patients were included in the study, 70 in the leflunomide group and 40 in the cyclophosphamide group. In their study, the therapy of leflunomide and prednisone induced complete remission in $21 \%$ of patients and partial remission in $52 \%$ within 6 months. Thus, $73 \%$ of leflunomide-treated patients had a significant improvement in their diseases, a response that 
was essentially identical to cyclophosphamide treatment [20]. Besides, some patients in their study went through repeat biopsy to confirm the efficacy of leflunomide. Repeat biopsies performed in patients treated with leflunomide and prednisone and blindly reviewed showed that the decrease in AI paralleled the improving clinical indices.

In the current study, the total remission rate was similar to that in Wang's study. Seventy-nine percent of leflunomidetreated patients achieved partial or complete remission at the end of the study. After the 6-month treatment, proteinuria, serum albumin, and complement 3 level were significantly improved; serum creatinine stabilized or decreased. Significant decreases were seen in SLEDAI and anti-dsDNA antibody titers in the leflunomide group.

Although this project is a small-scale study, a number of useful observations could be made from this study. The recommended dose of leflunomide in patients with rheumatoid arthritis is $20 \mathrm{mg}$ daily [13], but the recommended dose of leflunomide is unclear in LN. The dose of leflunomide used in previous studies was from 20 to $50 \mathrm{mg}$ daily. In this study, the dose of leflunomide is small when compared to that of the previous study (20 mg daily vs $30 \mathrm{mg}$ daily) [20]. Though the dose of leflunomide in this study is lower, there is no significant difference in efficacy. Our study showed that low-dose leflunomide is also safe and cost-effective compared with cyclophosphamide. The results suggested that low-dose leflunomide could be effective in the treatment of proliferative LN patients. In the current study, adverse events were common in both groups and the there was no significant difference between them. Major adverse events observed in the patients treated with leflunomide were similar to those in the cyclophosphamide group. We noticed that the percentage of patients with amenorrhea was lower in the leflunomide group compared with that of the cyclophosphamide group. This suggests that leflunomide might be safer than cyclophosphamide in the treatment of lupus nephritis.

There are some limitations in our study. First, though the study was an open, multi-center, randomized controlled clinical trial, the number of patients enrolled in this study was still not large enough. The patients enrolled in this study were from the same race, so the results may not be generalized in other races. The study was not blinded due to the form of leflunomide and cyclophosphamide. Second, the number of patients enrolled is not big enough. Because the time of the study was limited, we did not include enough patients. Third, we did not observe the efficacy of leflunomide in the maintenance therapy in these patients with proliferative LN. Weneed to make long-term follow-up to these patients. Third, patients in our study have no biopsy after treatment to evaluate the pathological change.

\section{Conclusions}

In summary, low-dose leflunomide combined with glucocorticoids was effective in the induction treatment of proliferative lupus nephritis in Chinese patients, and the efficacy and safety was similar to cyclophosphamide. Leflunomide was generally well-tolerated; however, precautions are needed to be taken during the leflunomide treatment to monitor infections especially when larger dosage of steroids was used concomitantly. Long-term, randomized studies are needed to elucidate the efficacy and safety of leflunomide in treating lupus nephritis.

Acknowledgements Drugs were provided by Fujian Huitian Biological Pharmaceutical Co.,ltd. Thanks to all the members that participated in this clinical trial from multi-centers: nephrologists from Guizhou Provincial People's Hospital; Fuzhou General Hospital of Nanjing Military Command; The Second Hospital of Jilin University; Wuxi People's Hospital; the First Affiliated Hospital of Fujian Medical University, Fuzhou, China; The First Affiliated Hospital of Nanjing Medical University; Peking University Third Hospital; Xin Hua Hospital Affiliated to Shanghai Jiaotong University School of Medicine; The First Bethune Hospital of Jilin University; The First Affiliated Hospital of Nanchang University; The First Affiliated Hospital, College of Medicine, Zhejiang University; Zhongshan Hospital, Xiamen University; Shenjing Hospital of China Medical University.

Authorship contributions $\mathrm{MZ}$ and $\mathrm{CQ}$ analyzed and interpreted the patient data. YZ, JC, PL, LW, ZS, JW, CX, SW, GJ, MS, QC, JC, TG, and DL screened subjects into groups and did follow-up work. MZ was a major contributor in writing the manuscript. $\mathrm{ZN}$ designed the study and helped to revise the manuscript. All authors read and approved the final manuscript.

\section{Compliance with ethical standards}

The study was approved by the Chinese State Food and Drug Administration (2011L01968) and the ethics committees of Renji Hospital, School of Medicine, Shanghai Jiaotong University, and informed consent was obtained from each patient.

Disclosures None.

Open Access This article is distributed under the terms of the Creative Commons Attribution 4.0 International License (http:// creativecommons.org/licenses/by/4.0/), which permits unrestricted use, distribution, and reproduction in any medium, provided you give appropriate credit to the original author(s) and the source, provide a link to the Creative Commons license, and indicate if changes were made.

\section{References}

1. Yu C, Gershwin ME, Chang C (2014) Diagnostic criteria for systemic lupus erythematosus: a critical review. J Autoimmun 48-49: 10-13. https://doi.org/10.1016/j.jaut.2014.01.004

2. Mok CC, Kwok RC, Yip PS (2013) Effect of renal disease on the standardized mortality ratio and life expectancy of patients with systemic lupus erythematosus. Arthritis Rheum 65(8):2154-2160. https://doi.org/10.1002/art.38006 
3. Borchers AT, Leibushor N, Naguwa SM, Cheema GS, Shoenfeld Y, Gershwin ME (2012) Lupus nephritis: a critical review. Autoimmun Rev 12(2):174-194. https://doi.org/10.1016/j.autrev. 2012.08.018

4. Austin HA 3rd, Klippel JH, Balow JE, le Riche NG, Steinberg AD, Plotz PH, Decker JL (1986) Therapy of lupus nephritis. Controlled trial of prednisone and cytotoxic drugs. N Engl J Med 314(10):614 619. https://doi.org/10.1056/NEJM198603063141004

5. Contreras G, Pardo V, Leclercq B, Lenz O, Tozman E, O'Nan P, Roth D (2004) Sequential therapies for proliferative lupus nephritis. N Engl J Med 350(10):971-980. https://doi.org/10.1056/ NEJMoa031855

6. Houssiau FA, Vasconcelos C, D'Cruz D, Sebastiani GD, de Ramon GE, Danieli MG, Abramovicz D, Blockmans D, Cauli A, Direskeneli H, Galeazzi M, Gul A, Levy Y, Petera P, Popovic R, Petrovic R, Sinico RA, Cattaneo R, Font J, Depresseux G, Cosyns JP, Cervera R (2010) The 10-year follow-up data of the Euro-Lupus Nephritis Trial comparing low-dose and high-dose intravenous cyclophosphamide. Ann Rheum Dis 69(1):61-64. https://doi.org/10. 1136/ard.2008.102533

7. Chan TM, Li FK, Tang CS, Wong RW, Fang GX, Ji YL, Lau CS, Wong AK, Tong MK, Chan KW, Lai KN (2000) Efficacy of mycophenolate mofetil in patients with diffuse proliferative lupus nephritis. Hong Kong-Guangzhou Nephrology Study Group. Engl J Med 343(16):1156-1162. https://doi.org/10.1056/ NEJM200010193431604

8. Appel GB, Contreras G, Dooley MA, Ginzler EM, Isenberg D, Jayne D, Li LS, Mysler E, Sanchez-Guerrero J, Solomons N, Wofsy D, Aspreva Lupus Management Study G (2009) Mycophenolate mofetil versus cyclophosphamide for induction treatment of lupus nephritis. J Am Soc Nephrol 20(5):1103-1112. https://doi.org/10.1681/ASN.2008101028

9. Rovin BH (2009) Glomerular disease: lupus nephritis treatment: are we beyond cyclophosphamide? Nat Rev Nephrol 5(9):492-494. https://doi.org/10.1038/nrneph.2009.130

10. Hahn BH, McMahon MA, Wilkinson A, Wallace WD, Daikh DI, Fitzgerald JD, Karpouzas GA, Merrill JT, Wallace DJ, Yazdany J, Ramsey-Goldman R, Singh K, Khalighi M, Choi SI, Gogia M, Kafaja S, Kamgar M, Lau C, Martin WJ, Parikh S, Peng J, Rastogi A, Chen W, Grossman JM, American College of R (2012) American College of Rheumatology guidelines for screening, treatment, and management of lupus nephritis. Arthritis Care Res 64(6):797-808. https://doi.org/10.1002/acr.21664

11. Radhakrishnan J, Cattran DC (2012) The KDIGO practice guideline on glomerulonephritis: reading between the (guide)lines-application to the individual patient. Kidney Int 82(8):840-856. https:// doi.org/10.1038/ki.2012.280

12. Davis JM 3rd, Matteson EL, American College of R, European League Against R (2012) My treatment approach to rheumatoid arthritis. Mayo Clin Proc 87(7):659-673. https://doi.org/10.1016/j. mayocp.2012.03.011

13. Behrens F, Koehm M, Burkhardt H (2011) Update 2011: leflunomide in rheumatoid arthritis - strengths and weaknesses. Curr Opin Rheumatol 23(3):282-287. https://doi.org/10.1097/ BOR.0b013e328344fddb

14. Breedveld FC, Dayer JM (2000) Leflunomide: mode of action in the treatment of rheumatoid arthritis. Ann Rheum Dis 59(11):841849

15. Fragoso YD, Brooks JB (2015) Leflunomide and teriflunomide: altering the metabolism of pyrimidines for the treatment of autoimmune diseases. Expert Rev Clin Pharmacol 8(3):315-320. https:// doi.org/10.1586/17512433.2015.1019343

16. Remer CF, Weisman MH, Wallace DJ (2001) Benefits of leflunomide in systemic lupus erythematosus: a pilot observational study. Lupus 10(7):480-483. https://doi.org/10.1191/ 096120301678416033

17. Tam LS, Li EK, Wong CK, Lam CW, Szeto CC (2004) Doubleblind, randomized, placebo-controlled pilot study of leflunomide in systemic lupus erythematosus. Lupus 13(8):601-604. https://doi. org/10.1191/0961203304lu1067sr

18. Yang S, Xie L, Xue W, Yin A, Lu W (2013) Leflunomide plus oral prednisone in treatment of idiopathic membranous nephropathy: a retrospective clinical study of efficacy and safety. Nephrology 18(9):615-622. https://doi.org/10.1111/nep.12143

19. Lou T, Wang C, Chen Z, Shi C, Tang H, Liu X, Yin P, Yu X (2006) Randomised controlled trial of leflunomide in the treatment of immunoglobulin A nephropathy. Nephrology 11(2):113-116. https:// doi.org/10.1111/j.1440-1797.2006.00547.x

20. Wang HY, Cui TG, Hou FF, Ni ZH, Chen XM, Lu FM, Xu FF, Yu XQ, Zhang FS, Zhao XZ, Zhao MH, Wang GB, Qian JQ, Cai GY, Zhu TY, Wang YH, Jiang ZP, Li YN, Mei CL, Zou WZ, China Leflunomide Lupus Nephritis Study G (2008) Induction treatment of proliferative lupus nephritis with leflunomide combined with prednisone: a prospective multi-centre observational study. Lupus 17(7):638-644. https://doi.org/10.1177/0961203308089408

21. Tam LS, Li EK, Wong CK, Lam CW, Li WC, Szeto CC (2006) Safety and efficacy of leflunomide in the treatment of lupus nephritis refractory or intolerant to traditional immunosuppressive therapy: an open label trial. Ann Rheum Dis 65(3):417-418. https://doi. org/10.1136/ard.2005.044248

22. Cao H, Rao Y, Liu L, Lin J, Yang H, Zhang X, Chen Z (2015) The efficacy and safety of leflunomide for the treatment of lupus nephritis in Chinese patients: systematic review and meta-analysis. PLoS One 10(12):e0144548. https://doi.org/10.1371/journal.pone. 0144548

23. Hochberg MC (1997) Updating the American College of Rheumatology revised criteria for the classification of systemic lupus erythematosus. Arthritis Rheum 40(9):1725. https://doi.org/ 10.1002/1529-0131(199709)40:9\&1t;1725::AID-ART29\&gt;3.0. $\mathrm{CO} ; 2-\mathrm{Y}$

24. Weening JJ, D'Agati VD, Schwartz MM, Seshan SV, Alpers CE, Appel GB, Balow JE, Bruijn JA, Cook T, Ferrario F, Fogo AB, Ginzler EM, Hebert L, Hill G, Hill P, Jennette JC, Kong NC, Lesavre P, Lockshin M, Looi LM, Makino H, Moura LA, Nagata M, International Society of Nephrology Working Group on the Classification of Lupus N, Renal Pathology Society Working Group on the Classification of Lupus N (2004) The classification of glomerulonephritis in systemic lupus erythematosus revisited. Kidney Int 65(2):521-530. https://doi.org/10.1111/j.1523-1755. 2004.00443.x

25. Austin HA 3rd, Muenz LR, Joyce KM, Antonovych TT, Balow JE (1984) Diffuse proliferative lupus nephritis: identification of specific pathologic features affecting renal outcome. Kidney Int 25(4): 689-695

26. Wiacek R, Kolossa K, Jankowski T, Jeka S, Karmowski A, Karmowski M, Gworys B (2012) The efficacy and safety of leflunomide in patients with active rheumatoid arthritis. Adv Clin Exp Med 21(3):337-342

27. $\mathrm{Xu} X$, Blinder L, Shen J, Gong H, Finnegan A, Williams JW, Chong AS (1997) In vivo mechanism by which leflunomide controls lymphoproliferative and autoimmune disease in MRL/MpJlpr/lpr mice. J Immunol 159(1):167-174

28. He C, Lu X, Yan Z, Wu M, Liu S, Yu Y, Luo P (2012) Therapeutic effect of leflunomide on the development of experimental lupus nephritis in mice. Rheumatol Int 32(3):633-638. https://doi.org/ $10.1007 / \mathrm{s} 00296-010-1630-\mathrm{z}$ 


\section{Affiliations}

Minfang Zhang ${ }^{1} \cdot$ Chaojun $\mathrm{Qi}^{1} \cdot$ Yan Zha ${ }^{2} \cdot$ Jian Chen ${ }^{3} \cdot$ Ping Luo $^{4} \cdot$ Li Wang $^{5} \cdot$ Zhuxing Sun $^{6} \cdot$ Jianxin Wan ${ }^{7}$. Changying Xing ${ }^{8}$. Song Wang ${ }^{9} \cdot$ Gengru Jiang $^{10} \cdot$ Mindan Sun ${ }^{11} \cdot$ Qinkai Chen ${ }^{12} \cdot$ Jianghua Chen $^{13} \cdot{\text { Detian } \mathrm{Li}^{14}}^{14}$ Tianjun Guan ${ }^{15} \cdot$ Zhaohui Ni $^{1}$

1 Department of Nephrology, Renji Hospital, School of Medicine, Shanghai Jiaotong University, Shanghai, China

2 Department of Nephrology, Guizhou Provincial People's Hospital, Guiyang, Guizhou, China

3 Department of Nephrology, Fuzhou General Hospital of Nanjing Military Command Area, Fuzhou, China

4 Department of Nephrology, The Second Hospital of Jilin University, Jilin, China

5 Department of Nephrology, Sichuan Provincial People's Hospital, Chengdu, Sichuan, China

6 Department of Nephrology, Wuxi People's Hospital, Wuxi, China

7 Department of Nephrology, First Affiliated Hospital of Fujian Medical University, Fuzhou, China

8 Department of Nephrology, The First Affiliated Hospital of Nanjing Medical University, Nanjing, China
9 Department of Nephrology, Peking University Third Hospital, Peking, China

10 Department of Nephrology, Xin Hua Hospital, Shanghai Jiaotong University School of Medicine, Shanghai, China

11 Department of Nephrology, First Hospital of Jilin University, Jilin, China

12 Department of Nephrology, The First Affiliated Hospital of Nanchang University, Nanchang, Jiangxi, China

13 Department of The Kidney Disease Center, The First Affiliated Hospital, College of Medicine, Zhejiang University,

Hangzhou, Zhejiang, China

14 Department of Nephrology, Shengjing Hospital of China Medical University, Shenyang, Liaoning, China

15 Department of Nephrology, Zhongshan Hospital, Xiamen University, Xiamen, Fujian, China 